\title{
The Chromatic Index of Graphs with a Spanning Star
}

Mike Plantholt

UNIVERSITY OF MICHIGAN

ANN ARBOR, MICHIGAN

\section{ABSTRACT}

Vizing's Theorem states that any graph $G$ has chromatic index either the maximum degree $\Delta(G)$ or $\Delta(G)+1$. If $G$ has $2 s+1$ points and $\Delta(G)=2 s$, a well-known necessary condition for the chromatic index to equal $2 s$ is that $G$ have at most $2 s^{2}$ lines. Hilton conjectured that this condition is also sufficient. We present a proof of that conjecture and a corollary that helps determine the chromatic index of some graphs with $2 s$ points and maximum degree $2 s-2$.

\section{INTRODUCTION}

A line-coloring of a graph is an assignment of colors to its lines such that no two adjacent lines are assigned the same color. The chromatic index of a graph $G$ is the minimum number of colors used among all line-colorings of $G$ and is denoted by $\chi^{\prime}(G)$. Vizing [5] has shown that for any graph $G$, either $\chi^{\prime}(G)=\Delta(G)$ or $\chi^{\prime}(G)=\Delta(G)+1$. If $\chi^{\prime}(G)=\Delta(G)$, then $G$ is in Class 1; otherwise $G$ is in Class 2. In general, determining the class of a given graph $G$ is very difficult. However, if $G$ contains a spanning star, that is, one point of $G$ is adjacent to all others, the problem is more manageable. If $G$ has $2 s$ points, $s$ a positive integer, then $G$ is in Class 1 since it is a subgraph of $K_{2 s}$, which can be line-colored using $2 s-1$ colors (see Lemma 2 below). If $G$ has $2 s+1$ points, the problem is not quite as easy. Our object is to determine the chromatic index of such graphs.

In the discussion below, we follow [4] for all terminology and notation unless stated otherwise; for basic results on line-colorings see [1]. In addition, a "coloring" of a graph will always mean a line-coloring, while an " $n$-coloring" will be a coloring that uses only $n$ colors. Finally, once a graph $G$ is colored, we will say that the point $v$ of $G$ "misses" color $C$ (and, conversely, $C$ misses $v$ ) if no line assigned color $C$ is incident with $v$. 


\section{PROOF OF HILTON'S CONJECTURE}

Let $G$ be a graph with $2 s+1$ points, where $s$ is a positive integer, and suppose $\Delta(G)=2 s$. Since any set of independent lines of $G$ can have cardinality at most $s$, a necessary condition for $G$ to be in Class 1 is that $G$ have at most $2 s \cdot s=2 s^{2}$ lines. Hilton [3] conjectured that this condition is also sufficient. We now present a proof of that conjecture. We will need the following standard results [1].

Lemma 1. Every bipartite graph is in Class 1.

Lemma 2. The complete graph $K_{n}$ is in Class 1 for $n$ even and in Class 2 for $n$ odd.

Definition. Let $G$ be an arbitrary graph and let $H=\left(h_{1}, \ldots, h_{n}\right)$ be a nonincreasing sequence of non-negative integers. Sequence $H$ is said to be coloring-feasible for $G$ if there exists an $n$-coloring of the lines of $G$ for which the cardinalities of the resulting $n$ color classes are precisely $h_{1}, \ldots, h_{n}$.

Lemma 3. [2] Let $G$ be an arbitrary graph. If sequence $H=\left(h_{1}, \ldots, h_{n}\right)$ is coloring-feasible for $G$, then so is any sequence $H^{\prime}=\left(h_{1}^{\prime}, \ldots, h_{n}^{\prime}\right)$ such that

$$
\sum_{i=1}^{n} h_{i}^{\prime}=\sum_{i=1}^{n} h_{i} \text { and for } k=1, \ldots, n-1, \sum_{i=1}^{k} h_{i}^{\prime} \leq \sum_{i=1}^{k} h_{i}
$$

Remark. If $G$ can be $n$-colored, then by Lemma 3 there is an $n$-coloring of $G$ such that the cardinalities of any two color classes differ by at most one. Such a coloring is called an equitable coloring of $G$.

Lemma 4. Let $n$ be an odd integer. If $K_{n}$ is colored with $n$ colors, then each of the $n$ colors misses exactly one point, and each point misses exactly one color.

Proof. $K_{n}$ has $\left(\begin{array}{l}n \\ 2\end{array}\right)=n(n-1) / 2$ lines. In order to achieve an $n$ coloring, each color class must have cardinality $(n-1) / 2$, since it can be no more. So, each color misses exactly one point. Also, since each point has degree $n-1$, each point misses exactly one color.

We are now able to prove the main result. Recall that a star is a complete bipartite graph $K_{1, n}$.

Theorem. Let $G$ be a graph of odd order $2 s+1$ which contains a spanning star. Then $G$ is in Class 1 if and only if $G$ has at most $2 s^{2}$ lines.

Proof. Since $\Delta(G)=2 s$, it is clear from the previous discussion that we need only show the sufficiency. Also, note that removing lines from a graph 
cannot increase its chromatic index, so it suffices to show that $\chi^{\prime}(G)=2 s$ if $G$ has exactly $2 s^{2}$ lines (or, equivalently, $G$ is $K_{2 s+1}$ with exactly $s$ lines removed). To do so, we will write $G$ as the direct sum (also called the sum) of two factors of $G$, each with chromatic index $s$.

Case 1. $s$ is odd.

Let $v_{1}, \ldots, v_{m}$ be those points which have degree at least two in the complement $\bar{G}$. Writing $\bar{d}\left(v_{i}\right)$ for the degree of $v_{i}$ in $\bar{G}$, since $\sum_{i=1}^{m} \bar{d}\left(v_{i}\right) \geq$ $2 m$, it follows that $\left\{v_{1}, \ldots, v_{m}\right\}$ must be incident with at least $m$ distinct lines of $\bar{G}$. Also, since $\bar{G}$ contains only $s$ lines, $m \leq s$. Therefore, we can augment $\left\{v_{1}, \ldots, v_{m}\right\}$ to a set $L=\left\{v_{1}, \ldots, v_{m}, v_{m+1}, \ldots, v_{s+1}\right\}$ such that any line of $\bar{G}$ must be incident with a member of $L$. Also, note that any point not in $L$ has degree at most one in $\bar{G}$. Next, let $R$ be the set consisting of the $s$ points of $G$ not included in $L$, and denote this set by $R=\left\{w_{1}, \ldots, w_{s}\right\}$.

Define a spanning bipartite subgraph $B$ of $G$ such that two points are adjacent in $B$ if and only if they are adjacent in $G$ and one of them is in $L$, the other in $R$. So, $B$ is a subgraph of $K_{s+1, s}$ and hence $\Delta(B) \leq s+1$. If we let $J$ be the set of points of $B$ with degree $s+1$, then $J \subset R$. Without loss of generality, assume $J=\left\{w_{1}, w_{2}, \ldots, w_{j}\right\}$.

Next define a graph $Z$ by $Z=G-E(B)$. Note that if we let $X$ be the subgraph of $G$ induced by $L$, and $Y$ the subgraph of $G$ induced by $R$, then we merely have $Z=X \cup Y$. Also, since

(i) $X \subset K_{s+1}$ implies $X^{\prime}(X) \leq s$ by Lemma 2,

(ii) $Y=K_{s}$ implies $X^{\prime}(Y)=s$, and

(iii) $X$ and $Y$ are disjoint

we see that the lines of $Z$ can be colored with $s$ colors. Thus $G$ can be written as the direct sum of two graphs, $B$ and $Z$, where $\chi^{\prime}(B) \leq s+1$ (by Lemma 1) and $\chi^{\prime}(Z)=s$. Note that if $J$ is empty, then $\chi^{\prime}(B)=s$ by Lemma 1 , and we are done. So, suppose $J$ is nonempty (so $j \geq 1$ ). To complete the proof of Case 1 , we will remove $j$ lines from $B$, each one incident with a different point of $J$, and place them as the corresponding lines in $Z$, without increasing the chromatic index of $Z$.

First note that since each line of $\bar{G}$ is incident with a point of $L$, the edges of $\bar{G}$ are given by $E\left(K_{s+1, s}-E(B)\right) \cup E(\bar{X})$, where the points of $K_{s+1, s}$ and $B$ are identified in the obvious way. Also, since $\bar{d}\left(w_{i}\right) \leq 1$ for $i=1, \ldots, s$, the graph $K_{s+1, s}-E(B)$ contains exactly $s-j$ lines. Therefore $\bar{X}$ contains exactly $j$ lines. Since $X$, being a subgraph of $K_{s+1}$, is $s$-colorable, it follows from Lemma 3 that the equitable sequence $H=\left(h_{1}, \ldots, h_{s}\right)$, where

$$
h_{i}= \begin{cases}(s+1) / 2 & i=1, \ldots, s-j \\ (s-1) / 2 & i=s-j+1 \ldots, s\end{cases}
$$

is coloring-feasible for $X$.

Perform such a coloring of $X$. Since $j$ of the colors, call them $C_{1}, \ldots, C_{j}$, are 
assigned to only $(s-1) / 2$ lines each, we see that for any $i, i=1, \ldots, 22$, the color $C_{i}$ misses two point of $X$, call them $u_{i 1}$ and $u_{i 2}$.

Next, color $Y$ with the same $s$ colors used in the coloring of $X$, so that $C_{i}$ misses $w_{i}, i=1, \ldots, j$. This is possible by Lemma 4 and the symmetry of $Y$. Finally, add to $Z$ the lines $u_{i 1} \mathrm{w}_{i}$, for $i=1, \ldots, j$, and color them $C_{1}, \ldots, C_{j}$ respectively; then remove the corresponding $j$ lines from $B$ (these lines are guaranteed to be in $B$ since each of $w_{1}, \ldots, w_{j}$ had degree $s+1$ in $B$ and $\left.u_{i 1} \in L\right)$. Now we have $\Delta(B)=s$, so that $\chi^{\prime}(B)=s$ by Lemma 1 , while $\chi^{\prime}(Z)$ is still $s$ by the construction above. Therefore, since $G$ is the direct sum of $B$ and $Z$, we have $\chi^{\prime}(G)=2 s$.

Case 2. $s$ is even.

The basic idea of the proof of this case is essentially the same as that of Case 1 . We will again write $G$ as the direct sum of two graphs, each with chromatic index equal to $s$. We proceed by using induction on $s$. It is easily verfied that the theorem is true for small $s$. Suppose, then, that the theorem holds for graphs with $2 i+1$ points, $i=1,2, \ldots, s-1$. We now require another lemma.

Lemma 5. Let $G^{\prime}$ be an arbitrary graph with $2 s+1$ points, $s$ even, and exactly $s$ lines. Then one of the following two conditions holds.

(i) There exists a set of $s+1$ points of $G^{\prime}$, call it $L$, such that the subgraph of $G^{\prime}$ induced by $L$ has at least $s / 2$ lines, every line of $G^{\prime}$ is incident with a point of $L$, and every point of degree greater than one in $G^{\prime}$ is an element of $L$.

(ii) There exists a set $L$ of $s+1$ points of $G^{\prime}$ such that the subgraph of $G^{\prime}$ induced by $L$ has exactly $s / 2$ lines, and any point with degree greater than one in $G^{\prime}$ is included in $L$.

Proof of Lemma 5. Order the points of $G^{\prime}$ by nonincreasing degree and call them $u_{1}, \ldots, u_{2 s+1}$. Note that $\sum_{i=1}^{2 s+1} d\left(u_{i}\right)=2 s$. We consider two possibilities.

Case 5.1. $\sum_{i=1}^{s / 2} d\left(u_{i}\right) \geq s$.

Now $\left\{u_{1}, u_{2}, \ldots, u_{s / 2}\right\}$ is incident with at least $s / 2$ distinct lines. Let $t$ be the number of points of $G^{\prime}$ with degree greater than one, and let $r=\max \{t, s / 2\}$. Let $L=\left\{u_{1}, \ldots, u_{r}\right\}$. We will expand $L$ so that it has $s+1$ points satisfying (i).

Note that $r \leq \mathrm{s}$ and $L$ is at the moment incident with at least $r$ distinct lines. Add to $L$ a set of $s+1-r$ points so that any line of $G^{\prime}$ is incident with a point included in $L$. This is possible since $L$ previously covered all but at most $s-r$ lines. We can assume that it was possible to choose the newly added $s+1-r$ points so that each has nonzero degree in $G^{\prime}$, for otherwise 
the number of point of $G^{\prime}$ with nonzero degree is less than $s+1$, in which the case the set of points with nonzero degree could be arbitrarily expanded to satisfy (i). So, the sum of the degrees of the points of $L$ is at least $s+s / 2+1$. Therefore, since $G^{\prime}$ has only $s$ lines, the subgraph of $G^{\prime}$ induced by the $s+1$ points in $L$ has at least $s / 2+1$ lines, and so property (i) is satisfied.

Case 5.2. $\sum_{i=1}^{s / 2} d\left(u_{i}\right)<s$.

Here, for $j>s / 2$, we have $d\left(u_{i}\right) \leq 1$. Let $L=\left\{u_{1}, \ldots, u_{s / 2}\right\}$. Now, since $\sum_{i=1}^{s / 2} d\left(u_{i}\right)<s$, the subgraph of $G^{\prime}$ induced by $L$ has less than $s / 2$ lines. We augment $L$ one point at a time as follows. Given $L$, let $v^{\prime}$ be a point of $G^{\prime}$ not yet in $L$ such that $d\left(v^{\prime}\right)=1$ and the number of lines in the subgraph of $G^{\prime}$ induced by $L \cup v^{\prime}$ is a maximum over all $L \cup v, v$ not in $L$. Then add $v^{\prime}$ to $L$.

Continue this procedure until $L$ induces exactly $s / 2$ lines in $G^{\prime}$. This is possible since we add at most one line to the induced subgraph for each additional point placed in $L$, and the algorithm assures that we get $s / 2$ lines induced with $|L|<s+1$, since $\sum_{i=1}^{s / 2} d\left(u_{i}\right) \geq s / 2$.

Once $L$ induces exactly $s / 2$ lines in $G^{\prime}$ we begin adding points to $L$ without increasing the induced number of lines. We claim that we can continue this process until $L$ contains $s+1$ points. For, suppose we reach a stage where no point can be added to $L$ without increasing the induced number of lines. Then each point of $G^{\prime}$ not yet included in $L$ has degree one, and no line of $G^{\prime}$ can have both its incident points in $V\left(G^{\prime}\right)-L$. Thus, there are at most $s$ points of $G^{\prime}$ not yet included in $L$, so that $L$ already includes at least $s+1$ elements.

After augmenting $L$ to $s+1$ points as outlined above, we note that it satisfies the conditions of property (ii), so that the proof of Lemma 5 is complete.

We now return to the proof of Case 2 of the theorem. Recall that the graph $G$ has $2 s+1$ points, $s$ even, and $\bar{G}$ has exactly $s$ lines. By Lemma 5 , we have two possibilities.

Case 2.1. There is a set of $s+1$ points, $L=\left\{v_{1}, \ldots, v_{s+1}\right\}$, such that the subgraph of $\bar{G}$ induced by $L$ has at least $s / 2$ lines, every line of $\bar{G}$ is incident with a point of $L$, and every point of $G$ with degree greater than one in $\bar{G}$ is included in $L$.

Let $R$ be the set of all points of $G$ not included in $L$. Label $R=\left\{w_{1}, \ldots, w_{s}\right\}$. Let $X$ denote the subgraph of $G$ induced by $L$, and $Y$ the subgraph of $G$ induced by $R$. As before, let $B$ be the spanning bipartite subgraph of $G$ whose lines are all $v_{t} w_{r}, 1 \leq t \leq s+1,1 \leq r \leq s$, such that $v_{t} w_{r}$ is a line in $G$. Again write $Z$ for $X \cup Y$. Note that $\chi^{\prime}(Z)=\max \left\{\chi^{\prime}(X)\right.$, $\left.\chi^{\prime}(Y)\right\}$. But $Y=K_{s}, X^{\prime}(Y)=s-1$ by Lemma 2; on the other hand, $X$ is $K_{s+1}$ minus at least $s / 2$ lines, so by the induction hypothesis, $X^{\prime}(X) \leq s$. Therefore, $Z$ has chromatic index at most $s$. 
Next, since $B$ is bipartite and $\Delta(B)=s+1$, we see that $\chi^{\prime}(B)=s+1$ by Lemma 1 . Again, let $j$ be the number of points with degree $s+1$ in $B$. By the construction of $L$, we have $s / 2 \leq j \leq s$, and these $j$ points are all in the set $R$. Assume, without loss of generality, that these $j$ points are the set $J=\left\{w_{1}, \ldots, w_{j}\right\}$. Once again we seek to remove $j$ lines from $B$, one incident with each point of $J$, and replace the corresponding lines in $Z$ without causing the chromatic index of $Z$ to become greater than $s$.

First note that $X$ is merely $K_{s+1}$ minus $j$ lines, since $G$ is $K_{2 s+1}$ minus $s$ lines, $Y$ is $K_{s}$, and $B$ is $K_{s+1, s}$ minus $s-j$ lines. So, by the induction hypothesis and Lemma 3 , there is an equitable $s$-coloring of the lines of $X$ with color-cardinality sequence $H=\left(h_{1}, \ldots, h_{s}\right)$ where $h_{i}=s / 2$ for $i \leq s-(j-s / 2)$ and $h_{i}=s / 2-1$ otherwise. Let $k=s-(j-s / 2)$. Then the corresponding colors $C_{1}, \ldots, C_{k}$ miss one point of $X$ each, while the colors $C_{k+1}, \ldots, C_{s}$ each miss three points of $X$.

Now consider the graph $Y$ (which is merely $K_{s}$ ). Since the color-cardinality sequence $(s / 2, \ldots, s / 2,0)$ of length $s$ is coloring-feasible for $Y$, so is the equitable sequence $\left(t_{1}, \ldots, t_{s}\right)$ where $t_{i}=s / 2$ for $i \leq s / 2$ and $t_{i}=s / 2-1$ otherwise. Note that since we are coloring $K_{s}$ with $s$ colors, each point of $Y$ is missing exactly one color. Using the same $s$ colors we used in coloring $X$, we are thus able to color $Y$ so that

0 points miss color $C_{i}$, for $i=1, \ldots, s / 2$,

2 points miss color $C_{i}$, for $i=s / 2+1, \ldots, s$.

In addition, using the symmetry of $K_{s}$, we can assume that in this coloring of $Y$, the points $w_{1}, w_{2}$ miss color $C_{k+1}, w_{3}$ and $w_{4}$ miss $C_{k+2}$, and so forth until $w_{2 j-s-1}$ and $w_{2 j-s}$ miss $C_{s}$, while the other $s-j$ points of $J$ miss $C_{s / 2+1}, \ldots, C_{k}$, respectively.

Finally, we add $i$ lines to $Z$ by making each of $w_{1}, \ldots, w_{j}$ in $Y$ adjacent to a point in $X$ missing the same color missed by $w_{i}$, and assigning each new line that color missing from its two incident points. Note that even though $w_{1}$ and $w_{2}$ miss the same color, we can join them to different points of $X$ since $C_{k+1}$ misses three points in $X$. A similar argument holds for $w_{3}$ and $w_{4}, \ldots, w_{2 j-s-1}$ and $w_{2 j-s}$. So we have added $j$ lines to $Z$, but $\chi^{\prime}(Z)$ is still at most $s$.

Now remove the corresponding $j$ lines from $B$. Since each of $w_{1}, \ldots, w_{j}$ is incident with one of these lines, we now have $\Delta(B)=s$, so that $X^{\prime}(B)=s$.

Therefore, since $G$ is the direct sum of $B$ and $Z$, we have $\chi^{\prime}(G)=2 s$, so that $G$ is in Class 1 .

Case 2.2. There exists a set of $s+1$ points $L=\left\{v_{1}, \ldots, v_{s+1}\right\}$ such that the subgraph of $\bar{G}$ induced by $L$ has exactly $s / 2$ lines, and any point of $\bar{G}$ not in $L$ has degree in $\bar{G}$ at most one.

Let $R=V(G)-L$, and label these points so that $R=\left\{w_{1}, \ldots, w_{s}\right\}$. Let $X$ denote the subgraph of $G$ induced by $L$, and let $Y$ denote the subgraph of $G$ 
induced by $R$. Let $B$ be a spanning bipartite subgraph of $G$, where the lines of $B$ are all $v_{t} w_{r}, 1 \leq t \leq s+1,1 \leq r \leq s$, such that $v_{t} w_{r}$ is a line in $G$. Finally, let $Z$ be $L \cup \mathrm{R}$.

Note that $\Delta(B)=s+1$. Let $j$ be the number of points with degree $s+1$ in $B$. Then, by construction $s / 2 \leq j \leq s$, and $B$ is $K_{s+1, s}$ with $s-j$ specific lines removed. Again, the points with degree $s+1$ in $B$ must all be contained in $R$. Without loss of generality, assume they are $w_{1}, \ldots, w_{j}$.

By the induction hypothesis, $X^{\prime}(X)=s$. Also, since $Y$ is a subgraph of $K_{s}$ and $s$ is even, $X^{\prime}(Y) \leq s-1$. Therefore, $X^{\prime}(Z)=s$. Once again we seek to remove $j$ lines from $B$, one each incident with $w_{1}, \ldots, w_{j}$, and add the corresponding lines to the graph $Z$ without increasing the chromatic index of $Z$.

Since $B$ is $K_{s+1, s}$ minus $s-j$ lines and $X$ is $K_{s+1}$ minus $s / 2$ lines, $Y$ must be $K_{s}$ minus $j-s / 2$ lines. Since any point of $R$ has degree at most one in $G$, the $(j-s / 2)$ lines of $\bar{Y}$ are independent and thus are incident with exactly $(2 j-s)$ points of $Y$. Since all these points must then have degree $s+1$ in $B$ (again by construction, since no point of $R$ has degree greater than one in $\bar{G}$ ), we can assume these points are $w_{1}, \ldots, w_{2 j-s}$.

Since $X$ is $K_{s+1}$ minus exactly $s / 2$ lines, we can color $X$ with $s$ colors by the induction hypothesis. We do so, naming the colors $C_{1}, \ldots, C_{s}$. Note that each color misses exactly one point in $X$, since each color must appear $s / 2$ times.

Next, take any equitable $s$-coloring of $Y$, using the same $s$ colors as for $X$. Since this coloring is equitable, $j$ of the colors are missing exactly 2 points of $Y$ each, while the other $s-j$ colors do not miss any points of $Y$. Renaming the colors within $Y$, if necessary, we have the colors $C_{1}, \ldots, C_{j}$ missing two points of $Y$ each. Recalling that the degree in $Y$ of $w_{i}$ is $s-2$ for $i \leq 2 j-s$ and $s-1$ otherwise, we note that the points $w_{1}, \ldots, w_{2 j-s}$ are each missing exactly 2 colors, while the points $w_{2 j-s+1}, \ldots, w_{s}$ each miss 1 color. Therefore, associate with each point $w_{i}$ of $Y$ the set $W_{i}$ consisting of its 1 or 2 missing colors. By the reasoning above, the $2(s-j)$ sets $W_{2 j-s+1}, \ldots, W_{s}$ each contain one element. Pair these off into $s-j$ pairs of one-element sets and take the union within the individual pairs, obtaining $s-j$ sets of cardinality one or two.

Suppose for the time being that each of the unions results in a set with 2 elements. Combining these $s-j$ sets with $W_{1}, \ldots, W_{2 j-s}$ we get $j$ sets, each with two elements, and each element appearing in exactly two sets. Thus, the union of any $k$ sets contains at least $k$ distinct elements; therefore, by Hall's theorem [4, p. 53] there is a system of distinct representatives (SDR) for the sets. If the supposition above that the unions of the pairs of singleton sets always results in a set of cardinality two does not hold, an SDR can be obtained in a similar fashion-if any union results in a set of cardinality one, assign that set its element as a representative, and show the existence of an SDR for the two-element sets by the method above.

We now have a system of distinct representatives, each representative being a color associated through its set with a point of $Y$ missing that color. So we obtain a set of $j$ distinct points, each missing a particular associated 
color, no two associated colors the same, and $w_{1}, \ldots, w_{2 j-s}$ are in this set of $j$ points. Thus, renaming the points in $Y$ if necessary, we can assume the chosen points are $w_{1} \ldots, w_{j}$.

To finish the proof, we now add to $Z$ the lines from each of $w_{1}, \ldots, w_{j}$ to the point of $X$ missing the associated representative color, and assign the mutual missing color to that line. Then remove the lines corresponding to the $j$ lines added to $Z$ from the graph $B$.

Now $\Delta(B)=s$, so that $\chi^{\prime}(B)=s$; however, we still have $\chi^{\prime}(Z)=s$. Therefore, since $G$ is the direct sum of $B$ and $Z$, we obtain $\chi^{\prime}(G)=2 s$.

Corollary. Let $G$ be a graph with $2 s+2$ points and maximum degree $2 s$. If there is a point $v$ of $G$ such that $G-v$ has exactly $2 s^{2}$ lines, then $G$ is in Class 1.

Proof. By the previous theorem, $G-v$ can be $2 s$-colored. In such a coloring of $G-v$, each color misses exactly one point. Then to each line $v w_{i}$ incident with $v$ we can assign any color missing from $w_{i}$ in the $2 s$-coloring of $G-v$. Since each color previously missed only one point of $G-v$, no two lines incident with $v$ are assigned the same color, so that we have obtained a $2 s$-coloring of the graph $G$.

A connected graph $G$ is $\rho$-critical if $G$ is in Class $2, \Delta(G)=\rho$, and $G-e$ is in Class 1 for any edge $e$ of $G$. Much work has been done on critical graphs [1]. Our main theorem gives an infinite family of such graphs since it can be restated as follows.

Theorem. A graph of odd order $2 s+1$ is $2 s$-critical if and only if it has exactly $2 s^{2}+1$ lines.

\section{ACKNOWLEDGMENT}

I would like to thank Professor Frank Harary for his many helpful suggestions during the preparation of this paper.

\section{References}

[1] S. Fiorini and R. J. Wilson, Edge-Colorings of Graphs. Pitman, London (1977).

[2] J. Folkman and D. R. Fulkerson, Edge colorings in bipartite graphs. Combinatorial Mathematics and Its Applications. Edited by R. C. Bose and T. A. Dowling. University of North Carolina Press, Chapel Hill (1969) 561-577. 
[3] A. J. W. Hilton, Definitions of criticality with respect to edge-coloring. J. Graph Theory. 1 (1977) 61-68.

[4] F. Harary, Graph Theory. Addison-Wesley, Reading, MA (1969).

[5] V. G. Vizing, On an estimate of the chromatic class of a $p$-graph (Russian) Diskret. Analiz 3 (1964) 25-30. 